\section{A clinico-molecular predictor identifies follicular lymphoma patients at risk of early transformation after first-line immunotherapy}

Follicular lymphoma (FL) usually follows an indolent course and responds well to initial treatment. Overall survival (OS) has improved after the introduction of rituximab, ${ }^{1}$ yet most patients experience multiple relapses and die from lymphoma. For some patients, relapses are associated with transformation to aggressive disease with histological features of diffuse large B-cell lymphoma (DLBCL) and markedly inferior OS., ${ }^{2,3}$ There is no robust method for assessment of transformation risk at FL diagnosis. Prospective identification of high transformation risk would enable selection of patients for future treatments aiming to postpone or prevent transformation. We present a novel clinico-molecular predictor of transformation risk in rituximab-treated FL, based on a gene expression score capturing deregulation of the nuclear factor-kappa B (NF-кB) pathway.

Few gene expression signatures have been associated with transformation risk in FL, and none have been tested in patients treated with rituximab. In Gentles et al. ${ }^{4}$ an expression signature composed of modules of embryonic stem cell genes was associated with transformation risk. Brodtkorb et al..$^{5}$ found six gene expression signatures capturing deregulation of the NF- $\mathrm{KB}$ pathway and predicting transformation in the pre-rituximab era. NF- $\kappa B$ signaling is implicated in the pathogenesis of multiple B-cell lymphomas, including DLBCL.,

We used a novel cohort of 82 FL patients receiving firstline rituximab to investigate and further develop the expression-based transformation predictors described in Brodtkorb et al. ${ }^{5}$ The patients participated in two prospective randomized trials testing first-line rituximab with or without interferon- $\alpha .^{8,9}$ Gene expression and copy number profiles were obtained from $92 \mathrm{FL}$ biopsies from these patients (Online Supplementary Methods). Biopsies were obtained prior to first-line treatment for FL and prior to any transformation event.

Table 1 summarizes patients' clinical characteristics. All patients needed treatment at inclusion due to symptomatic FL. The proportion of patients in the Follicular Lymphoma International Prognostic Index (FLIPI) risk groups was representative for FL patients in general. Most patients were included at diagnosis of FL; a minority had been observed without treatment (Online Supplementary Figure S1). Median follow-up time from diagnosis of FL was 10.3 years (range: $0.5-26.0$ years). By the end of follow up, 24 patients $(29 \%)$ had not received new treatment and 21 of these were still alive. Overall survival at ten years was $77 \%$ [95\% confidence interval (CI): $66-85 \%$ ] (Figure 1A). The average annual transformation rate was $2.3 \%$ for the first ten years, and 21 patients $(26 \%)$ were diagnosed with histological transformation to DLBCL (Figure 1B). No additional patients fulfilled the clinical criteria for transformation, ${ }^{2}$ reflecting a high biopsy frequency at relapse in the participating study centers. Overall survival at ten years after transformation was 35\% (95\%CI: 13-60\%) (Figure 1C).

To explore the robustness of the six NF- $\mathrm{kB}$ deregulation signatures, we compared those in the current cohort and a previously published cohort consisting of $75 \mathrm{FL}$ biopsies from 44 patients treated with traditional chemotherapy $^{5}$ (Online Supplementary Methods, Online Supplementary Tables $S 1$ and S2, and Online Supplementary Figures S2-S4). For completeness, we considered all 14 deregulation signatures involving downstream targets of
Table 1. Clinical characteristics of the patients in the rituximab cohort. For median observation time, numbers in parentheses indicate the range.

\begin{tabular}{lc}
\hline Patients' characteristics & Observations ( $\mathbf{n}=\mathbf{8 2})$ \\
Year of diagnosis & $1989-2008$ \\
Mean age, years (IQR) & $54(28-82)$ \\
Sex (M:F) & $1: 1$ \\
FLIPI score & \\
Low (0-1), n (\%) & $25(30 \%)$ \\
Intermediate (2), n (\%) & $35(43 \%)$ \\
High $(>3), n(\%)$ & $22(27 \%)$ \\
\hline
\end{tabular}

Grade

Grade 1-2, n (\%) $\quad 76(93 \%)$

Grade 3a, n (\%)

Transformation

Histological and clinical, $\mathrm{n}(\%) \quad 21(26 \%)$

No transformation, $\mathrm{n}(\%)$

$61(74 \%)$

Median observation time (months)

All patients $(\mathrm{n}=82)$

$124(6-309)$

Alive at end of follow up $(n=62)$

Dead at end of follow up ( $\mathrm{n}=20)$

137 (79-309)

With transformation $(\mathrm{n}=21)$

Without transformation $(\mathrm{n}=61)$

$132(6-309)$

IQR: interquartile range; M: male; F: female; FLIPI: Follicular Lymphoma International Prognostic Index; n: number.

the NF- $\mathrm{KB}$ pathway described in Brodtkorb et al..$^{5}$ A score is calculated for each signature and biopsy by averaging the expression values of the genes in the signature. The scores were highly consistent across the patient cohorts, suggesting that they represent robust molecular traits of FL (Online Supplementary Table S1 and Online Supplementary Figures S2-S4). Among these, the BTK score measured in pre-treatment FL biopsies was associated with transformation in the rituximab-treated cohort (Student $t$-test, $P=0.039$ ) (Online Supplementary Table S3), validating the previous findings in the pre-rituximab era. A binary BTK score found by thresholding the score on its median was associated with transformation (Fisher's exact test, $P=0.022$ ) (Online Supplementary Figure S5), also after adjustment for FLIPI (Logistic regression, $P=0.019$ ). Among patients with high BTK score, the median time from detecting a high score to transformation was 51 months (range: 7-185 months); a similar result was found in the chemotherapy-treated cohort (Online Supplementary Figure S6). The binary BTK score was associated with time to transformation (Log rank test, $P=0.035)$ and time to new treatment (Log rank test, $P=0.050$ ) (Figure 1D and E). It was also correlated to the expression of the MKI67 gene, suggesting an association to increased proliferation in FL (Online Supplementary Figure S7).

Follicular Lymphoma International Prognostic Index risk groups (low, intermediate, high) were associated with time to transformation (Log rank test, $P=0.041$ ), but not with time to new treatment (Online Supplementary Figure $S 8 A$ and $B$ ). The binary BTK score and FLIPI were associated with time to transformation in a bivariate linear model [Cox's proportional hazards model, $P=0.029$; Hazard Ratio (HR) 3.18, 95\%CI: $1.15-8.74$, and $P=0.021$, HR 1.76, 95\%CI: 1.11-2.78, respectively]. Considering all combinations of FLIPI (low, intermediate or high) and the 
BTK score (low or high) in a multivariate interaction model, we defined a BTK-FLIPI score with six levels. This score was significantly associated with time to transformation (Log rank test, $P<0.0001$ ), but not with time to new treatment (Online Supplementary Figure S8C and D). Considering patients with high FLIPI and high BTK score versus all other patients, the association with time to transformation was highly significant (Log rank test, $P<0.0001$, HR 7.15, 95\%CI: 2.88-17.72) (Figure 2A) and the association with time to new treatment was also significant (Log rank test, $P=0.0031$, HR $2.59,95 \%$ CI: 1.35 4.99) (Figure 2B). Patients with high FLIPI and high BTK score (16\% of all patients) had inferior outcome with median time to new treatment of 21.7 months $(95 \% \mathrm{CI}$ : 14.3-42.6 months), compared to 73.6 months (95\% CI: 41.5-95.0 months) for other patients. Patients with high FLIPI and high BTK score had shorter time to transformation than patients with high FLIPI and low BTK score (Log rank test, $P=0.002$ ) (Figure $2 \mathrm{C}$ ). The risk of early progression was higher in the former patient group than in the latter (Log rank test, $P=0.015$ ) (Figure 2D). The proportion of patients with high BTK score was low in patients without progression, higher in patients progressing with indolent disease, and highest in patients progressing with transformation (Fisher's exact test, $P=0.015$ ) (Figure 3A). This trend was even stronger for the BTK-FLIPI score (Fisher's exact test, $P=0.0009$ ) (Figure 3B).
Expression of the $37 \mathrm{NF}-\kappa \mathrm{B}$ target genes constituting the BTK signature was compared in FL and de novo DLBCL. We included expression data from all available FL biopsies (rituximab cohort, $\mathrm{n}=92$ samples; chemotherapy cohort, $\mathrm{n}=75$ samples; $\mathrm{n}=167$ in total) and 150 de novo DLBCL biopsies. ${ }^{10}$ The highest BTK scores were found in de novo DLBCL. In FL, the BTK scores were highest in patients with later transformation (Online Supplementary Figure S9A). A multivariate analysis considering the first two principal components of the genes in the BTK signature revealed significant linear separation between FL with and without transformation (Linear discriminant analysis with permutation test, $P=0.004)$. Furthermore, a shift was seen towards de novo DLBCL for FL with later transformation (Hotelling's test, $P=0.003$ ) (Online Supplementary Figure S9B). Among all 14 NF- $\mathrm{KB}-$ related gene signatures, the BTK signature showed the most significant separation and shift towards DLBCL (Online Supplementary Table S4). Principal component analysis on a gene signature separating $A B C$ and $G C B$ subtypes of DLBCL ${ }^{11}$ revealed a similar shift, but not directed towards one specific subtype (Online Supplementary Figure S9C).

The BTK score involves NF- $\mathrm{KB}$ target genes correlated in expression with the gene Bruton tyrosine kinase ( $B T K)$, suggesting that aberrant B-cell receptor (BCR) signaling is an early event in transformation of FL. In con-
A

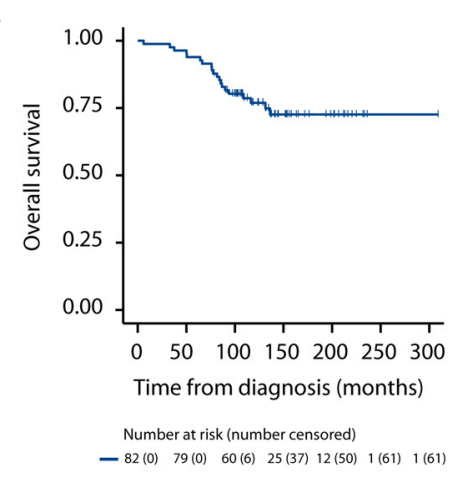

D

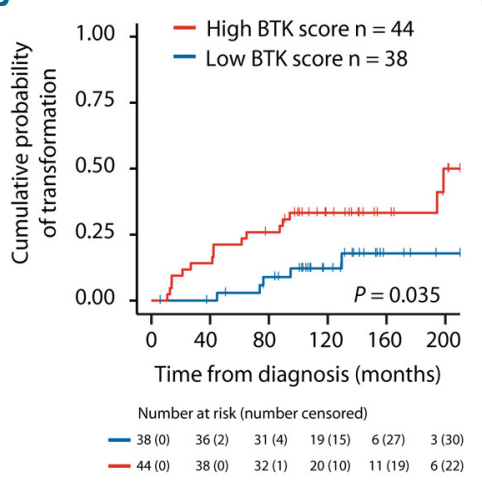

B

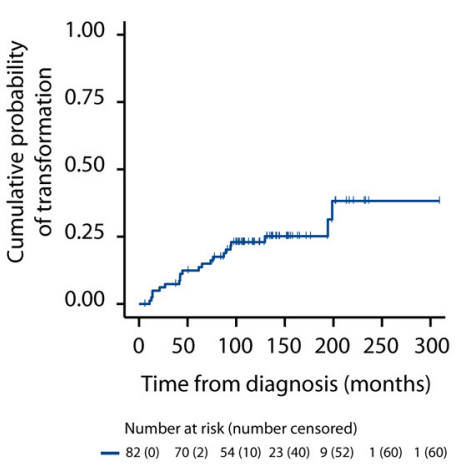

E

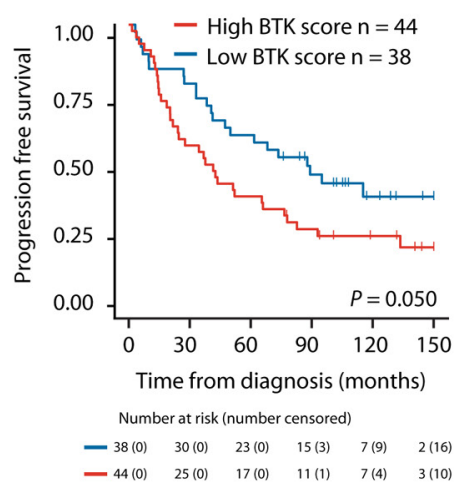

C

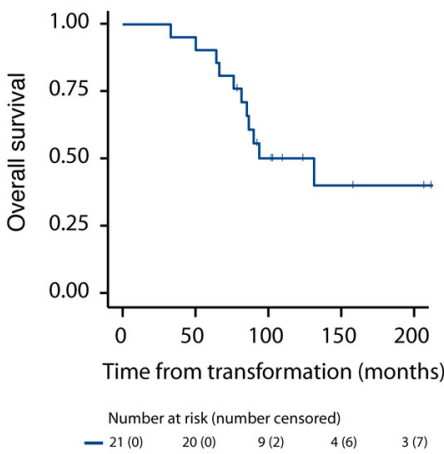

Figure 1. Kaplan-Meier plots of overall survival (OS) and cumulative probability of transformation in the rituximab cohort, and patient stratification according to the Bruton tyrosine kinase (BTK) score. (A) OS $(n=82)$. (B) Estimated cumulative probability of transformation over time $(n=82)$. (C) OS after transformation $(n=21)$. (D) Time to transformation in the group with high BTK score $(n=44)$ compared to the remainder $(n=38)(L o g$ rank test, $P<0.035 ; n=82$ patients). Among genes involved in NF-кB signaling with high cis-correlation between DNA copy number and gene expression values, BTK showed the highest cis-correlation (Online Supplementary Table S1 and Online Supplementary Figure S2). A BTK-signature was derived from a subset of NF-kB target genes (37 genes) (Online Supplementary Table S2) with expression values showing high correlation to BTK expression. For each patient, the binary BTK score was defined as high if the average expression of genes in the BTK-signature was above the median, and low if the average expression of BTK-signature genes was below the median. (E) Progression-free survival after rituximab in the group with high BTK score $(n=44)$ compared to the remainder $(n=38)($ Log rank test, $P=0.050$; $n=82$ patients). The end point for progression-free survival is start of new treatment for progression/relapse of lymphoma and death 
A

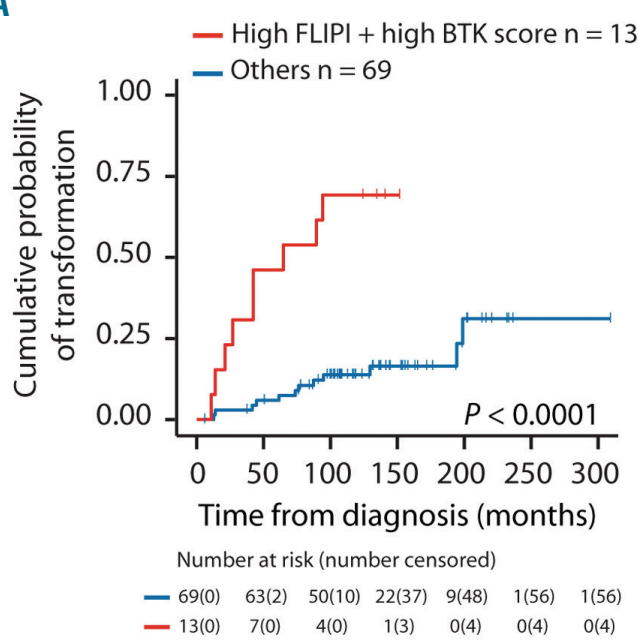

C

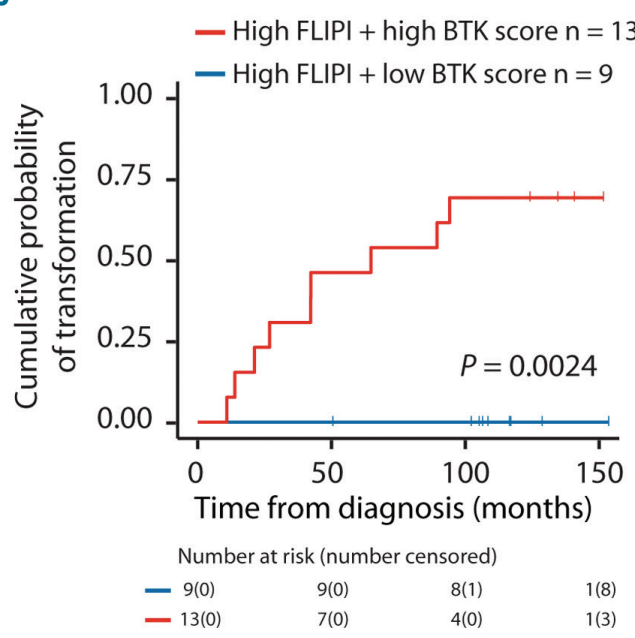

B

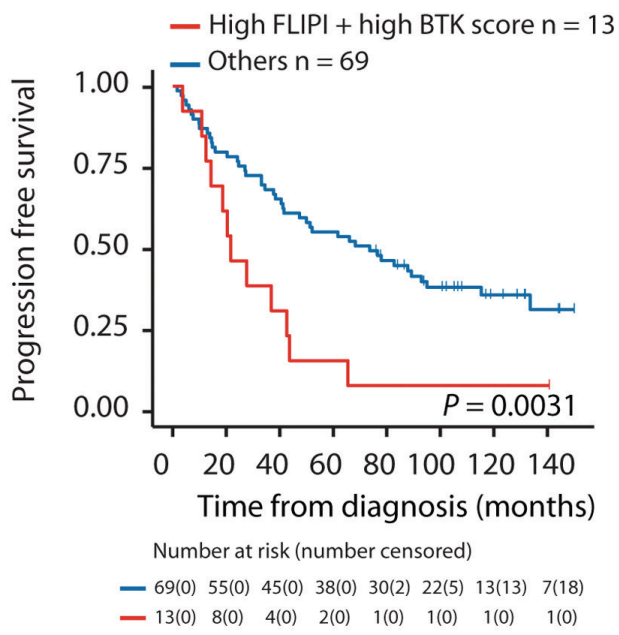

D

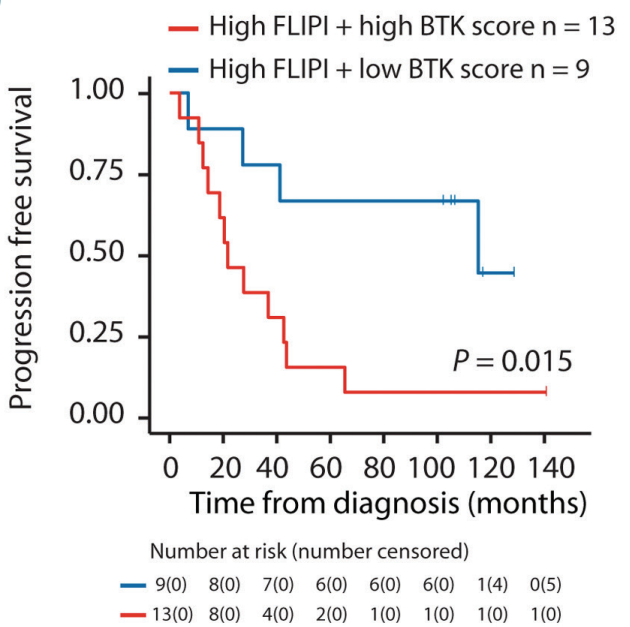

Figure 2. Kaplan-Meier plots according to the Bruton tyrosine kinase (BTK)-Follicular Lymphoma International Prognostic Index (FLIPI) predictor. (A) Time to transformation in the group with high FLIPI score and high BTK score $(n=13)$ compared to the remainder $(n=69)($ Log rank test, $P<0.0001$; $n=82$ patients). (B) Progression-free survival (PFS) after rituximab in the group with high FLIPI score and high BTK score $(n=13)$ compared to the remainder ( $\mathrm{n}=69)$ (Log rank test, $P=0.0031 ; n=82$ patients). (C) Time to transformation in the group with high BTK score and high FLIPI score compared to the group with high FLIPI and low BTK score (Log rank test: $P=0.0024 ; n=22$ patients). (D) PFS in the group with high BTK score and high FLIPI score compared to the group with high FLIPI and low BTK score (Log rank test, $P=0.015 ; n=22$ patients). In (B) and (D), the end point for PFS is start of new treatment for progression/relapse of lymphoma and death.

trast to several other B-cell malignancies, FL does not have constitutive $\mathrm{BCR}$ signaling., In protein signaling studies, chronic lymphocytic leukemia (CLL) and DLBCL tumor cells showed basal phosphorylation and activation of proteins involved in BCR signaling. Although no such basal activity was observed in $\mathrm{FL}^{12}$ the threshold for inducing BCR signaling in FL may be variable and more dependent on micro-environmental stimuli.

We propose the first combined clinical and gene expression score for identification of patients with high risk of transformation and early progression after rituximab monotherapy. Progression within two years after immunochemotherapy occurs in approximately $20 \%$ of FL patients, and is associated with poor OS. ${ }^{13}$ Rituximab monotherapy has demonstrated long-term outcome comparable to combinations with chemotherapy. ${ }^{14}$ Patients with high FLIPI and high BTK score accounted for $16 \%$ of FL patients and had median time to new treat- ment of less than two years. Among FLIPI high-risk patients, the patients with low BTK score showed good prognosis. The $\mathrm{m} 7$-FLIPI similarly reclassifies patients in the high-risk FLIPI group to the more favorable outcome group. ${ }^{15}$ Both models indicate that FL with high risk of transformation or treatment failure display specific molecular features already at diagnosis, and our findings strengthen the hypothesis that deregulation of the NF- $\mathrm{KB}$ pathway is an early manifestation of high risk in FL. While $\mathrm{m} 7$-FLIPI is based on molecular markers on the DNA level, the BTK-FLIPI score reflects gene expression, and may capture phenotypic variation not related to DNA sequence, including epigenetic modulation and properties of the tumor microenvironment. Mechanistic studies are needed to better understand the underlying biology of these prognostic models. In addition to molecular features, treatment decisions in FL will continue to be based on clinical information, and further develop- 
A

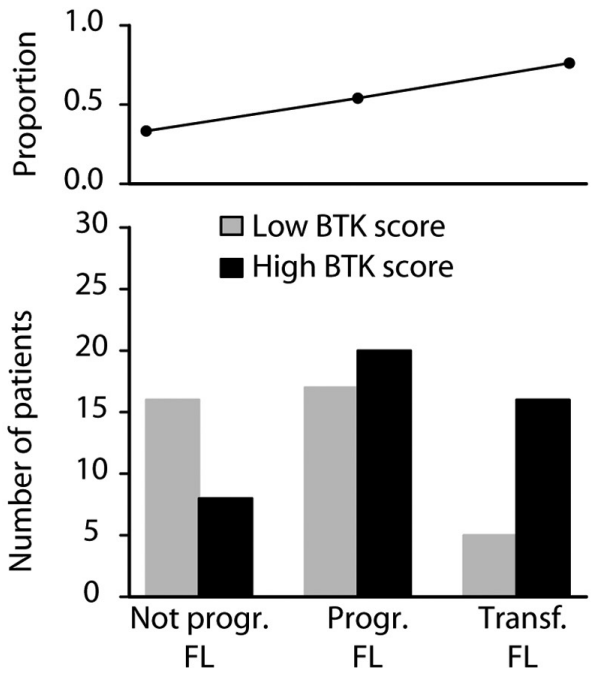

B

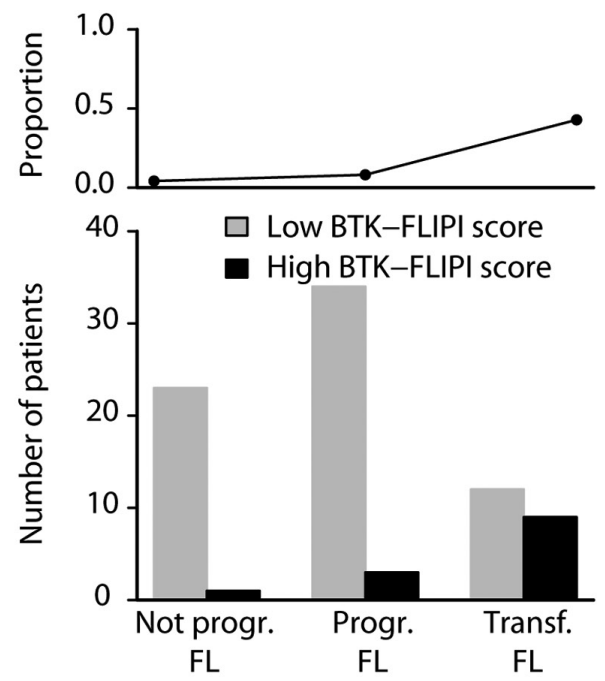

Figure 3. Distribution of follicular lymphoma (FL) patients according to disease outcome, Bruton tyrosine kinase (BTK) score and the BTK-Follicular Lymphoma International Prognostic Index (FLIPI) predictor. (A and B) Patients are divided into three groups according to disease outcome: 1) patients with progression of FL after primary treatment in the study and without any signs of transformation: No progr $(n=24) ; 2)$ patients progressing with indolent disease that needed new treatment: Progr ( $n=37)$; and 3) patients progressing with transformation to diffuse large B-cell lymphoma (DLBCL): Transf ( $n=21$ ). (A) Distribution of patients according to the three groups of disease outcome and Bruton tyrosine kinase (BTK) score. (Top) Proportion of patients with high BTK score. (Bottom) Number of patients with low BTK score (gray bar) and high BTK score (black bar) in FL cases without progression, cases progressing with indolent disease and cases progressing with transformation. (B) Distribution of patients according to the three groups of disease outcome and a binary BTK-Follicular Lymphoma International Prognostic Index (FLIPI) score where high BTK-FLIPI score is defined as a BTK score >median and FLIPI high (3-5), while low BTK-FLIPI are all remaining combinations of BTK score and FLIPI. (Top) Proportion of patients with high BTK-FLIPI score. (Bottom) Number of patients with low BTK-FLIPI score (gray bar) and high BTK-FLIPI score (black bar) in FL cases without progression, progression with indolent disease, and progression with transformation.

ment of clinico-molecular predictors may improve risk stratification and help guide treatment. In summary, we show that transformation is preceded by early deregulation of the NF- $\kappa B$ pathway. Identifying patients with high risk of transformation using the BTK-FLIPI score may aid treatment selection in FL.

Chloé B. Steen, ${ }^{1,2}$ Ellen Leich, June H. Myklebust, ${ }^{2,4}$ Sandra Lockmer, Jillian F. Wise, ${ }^{2,4}$ Björn E. Wahlin, Bjørn Østenstad, ${ }^{6}$ Knut Liestøl, ${ }^{1,7}$ Eva Kimby, Andreas Rosenwald, Erlend B. Smeland, ${ }^{2,4}$ Harald Holte, ${ }^{4,6}$ Ole Christian Lingjoerde ${ }^{1,4,8, *}$ and Marianne Brodtkorb $b^{2,4,6, "}$

${ }^{*} O C L$ and $M B$ contributed equally to this study.

${ }^{1}$ Department of Informatics, University of Oslo, Oslo, Norway; ${ }^{2}$ Department of Cancer Immunology, Institute for Cancer Research, Oslo University Hospital, Oslo, Norway; Institute of Pathology, University of Wurzburg and Comprehensive Cancer Centre Mainfranken, Germany; ${ }^{4}$ KG Jebsen Centre for B-Cell Malignancies, Institute for Clinical Medicine, University of Oslo, Oslo, Norwav; 'Division of Haematology, Department of Medicine at Huddinge, Karolinska Institute and University Hospital, Stockholm, Sweden; ${ }^{6}$ Department of Oncology, Oslo University Hospital, Oslo, Norway; 'Institute for Cancer Genetics and Informatics, Oslo University Hospital, Oslo, Norway; and ${ }^{8}$ Department of Cancer Genetics, Institute for Cancer Research, Oslo University Hospital, Oslo, Norway

Funding: this work was supported by the Research Council of Norway through its Centres of Excellence funding scheme (179571) and in part by Roche (EK, $A R$ ).

Acknowledgments: the gene expression data from LLMPP $191 \mathrm{FL}$ samples and 150 DLBCL samples) were obtained from published data from the Leukemia-Lymphoma Molecular Profiling Project headed by L.M. Staudt, National Cancer Institute, Rockville, MD.
Thanks to Theodora Nedeva, Tina Grieb and Inge Klier (Institute of Pathology, University of Wurzburg and Comprehensive Cancer Centre Mainfranken, Germany) for technical support.

Correspondence: OLE C. LINGJAERDE - ole@ifi.uio.no

MARIANNEBRODTKORB - meide@ous-hf.no

\section{doi:10.3324/haematol.2018.209080}

Information on authorship, contributions, and financial \& other disclosures was provided by the authors and is available with the online version of this article at www. haematologica.org.

\section{References}

1. Tan D, Horning SJ, Hoppe RT, et al. Improvements in observed and relative survival in follicular grade 1-2 lymphoma during 4 decades: the Stanford University experience. Blood. 2013;122(6):981-987.

2. Al-Tourah AJ, Gill KK, Chhanabhai M, et al. Population-based analysis of incidence and outcome of transformed non-Hodgkin's lymphoma. J Clin Oncol. 2008;26(32):5165-5169.

3. Link BK, Maurer MJ, Nowakowski GS, et al. Rates and outcomes of follicular lymphoma transformation in the immunochemotherapy era: a report from the University of Iowa/MayoClinic Specialized Program of Research Excellence Molecular Epidemiology Resource. J Clin Oncol. 2013;31(26):3272-3278.

4. Gentles AJ, Alizadeh AA, Lee SI, et al. A pluripotency signature predicts histologic transformation and influences survival in follicular lymphoma patients. Blood. 2009;114(15):3158-3166.

5. Brodtkorb M, Lingjærde OC, Huse K, et al. Whole-genome integrative analysis reveals expression signatures predicting transformation in follicular lymphoma. Blood. 2014;123(7):1051-1054.

6. Davis RE, Ngo VN, Lenz G, et al. Chronic active B-cell-receptor signalling in diffuse large B-cell lymphoma. Nature. 2010;463(7277):8892.

7. Saba NS, Liu D, Herman SE, et al. Pathogenic role of B-cell receptor signaling and canonical NF-kappaB activation in mantle cell lymphoma. Blood. 2016;128(1):82-92.

8. Kimby E, Jurlander J, Geisler C, et al. Long-term molecular remis- 
sions in patients with indolent lymphoma treated with rituximab as a single agent or in combination with interferon alpha-2a: a randomized phase II study from the Nordic Lymphoma Group. Leuk Lymphoma. 2008;49(1):102-112.

9. Kimby E, Ostenstad B, Brown P, et al. Two courses of four weekly infusions of rituximab with or without interferon-alpha2a: final results from a randomized phase III study in symptomatic indolent B-cell lymphomas. Leuk Lymphoma. 2015;56(9):2598-2607.

10. Wright G, Tan B, Rosenwald A, Hurt EH, Wiestner A, Staudt LM. A gene expression-based method to diagnose clinically distinct subgroups of diffuse large B cell lymphoma. Proc Natl Acad Sci U S A. 2003;100(17):9991-9996.

11. Davies AJ, Rosenwald A, Wright G, et al. Transformation of follicular lymphoma to diffuse large B-cell lymphoma proceeds by distinct oncogenic mechanisms. Br J Haematol. 2007;136(2):286-293.
12. Myklebust JH, Brody J, Kohrt HE, et al. Distinct patterns of B-cell receptor signaling in non-Hodgkin lymphomas identified by singlecell profiling. Blood. 2017;129(6):759-770.

13. Casulo C, Burack WR, Friedberg JW. Transformed follicular nonHodgkin lymphoma. Blood. 2015;125(1):40-47.

14. Lockmer S, Ostenstad B, Hagberg H, et al. Chemotherapy-Free Initial Treatment of Advanced Indolent Lymphoma Has Durable Effect With Low Toxicity: Results From Two Nordic Lymphoma Group Trials With More Than 10 Years of Follow-Up. J Clin Oncol. 2018; 36:(33)3315-3323.

15. Pastore A, Jurinovic V, Kridel R, et al. Integration of gene mutations in risk prognostication for patients receiving first-line immunochemotherapy for follicular lymphoma: a retrospective analysis of a prospective clinical trial and validation in a populationbased registry. Lancet Oncol. 2015;16(9):1111-1122. 